\title{
Are ECG abnormalities common in black Africans with heart failure? Results from the Heart of Soweto study
}

Geraldine Lee*, Melinda Carrington*, Karen Sliwa*\# and Simon Stewart*,\#

*Preventative Cardiology, Baker Heart Research Institute, Melbourne, Australia \#Department of Cardiology, Chris Hani Baragwanath Hospital, University of the Witwatersrand, Johannesburg, Republic of South Africa

Address for correspondence:

Professor Simon Stewart

Preventative Cardiology

Baker Heart Research Institute

PO Box 6492

St Kilda Rd Central

Melbourne Vic 8008

Australia

Email:

Simon.Stewart@baker.edu.au

ABSTRACT Background: The "Heart of Soweto" study is currently examining the pattern of cardiovascular disease (CVD) in Soweto, South Africa.

Methods:As part of a large clinical registry of CVD patients presenting to the Chris Hani Baragwanath Hospital in Soweto, a 12-lead ECG was performed on 91\% of “de novo" cases in 2006. Systematic analysis of the ECGs of heart failure patients was performed using detailed Minnesota coding.

Results: A total of 756 de novo patients with HF (57\% women, $88 \%$ Black African and mean age of $55 \pm 16$ years) had a I2-lead ECG. Overall, a major ECG abnormality was detected in $91 \%$ of cases consisting of ST segment changes, T wave changes, bundle branch blocks and arrhythmias. Minor abnormalities were noted in $97 \%$ of ECGs, such as axis deviation and high amplitude $\mathbf{R}$ waves. Left ventricular hypertrophy was evident in $15 \%$ (22\% in those with a dilated cardiomyopathy) and a bundle branch block in $8 \%$ of ECGs ( 1 \% in those with an ischemic cardiomyopathy).

Conclusion: Detailed examination of 12-lead ECGs of a large group of previously undiagnosed patients with HF in the Heart of Soweto study revealed many identifiable abnormalities. In addition to reconfirming the clinical utility of this cheap and practical diagnostic tool, these data highlight its potential role in screening for heart disease, particularly in low income communities.

\section{INTRODUCTION}

Heart failure (HF) is a deadly and debilitating clinical syndrome that is the final and common pathway of numerous cardiovascular disease (CVD) states. (I) Preliminary data from the Heart of Soweto study (one of the largest studies of heart disease in Africa) ${ }^{(2)}$ support the proposition that the phenomenon of epidemiologic transition and the subsequent emergence of more affluent forms of CVD seen in high income countries has broadened the pathways to the syndrome and increased the pool of affected individuals. ${ }^{(3)}$

In sub-Saharan Africa, HF has traditionally been caused by untreated rheumatic heart valvular disease, idiopathic and peripartum cardiomyopathy and hypertension. ${ }^{(4,5)}$

A particularly striking problem in this context is the usually late presentation of cases (i.e. where the antecedents to the syndrome have typically remained untreated, leading to clinically advanced forms of HF). The case in support of early detection and preventative treatment strategies is particularly strong in Soweto. However, those still imperfect strategies used to detect HF in high income countries, including systematic screening with brain natriuretic peptide assays ${ }^{(6)}$ and rapid access echocardiography ${ }^{(7)}$ may prove to be too costly and impractical in low to middle income countries such as South Africa.

A potentially cheap and useful diagnostic tool to detect HF in a relatively small number of adults living in large communities in low to middle income countries is the 12-lead electrocardiogram (ECG). Despite being "old" technology and commonly regarded as inferior to newer more costly, technologies, the ECG still plays an important role in the clinical assessment of patients with CVD. The ECG provides information on the heart's position within the chest, electrical disturbances and electrolyte disturbances. Furthermore, it shows the mass of cardiac muscle and indicates the presence of ischemic changes reflecting possible coronary artery disease (CAD) or ventricular dysfunction Many different tools have been developed to assess the 12-lead ECG including Cornell voltage, Sokolow-Lynn voltage and Romhilt-Estes indices. ${ }^{(8-10)}$ However, some authors have reported concerns with sensitivity and specificity. ${ }^{(9)}$ In particular, the voltage indices show 
differing results in determining $\mathrm{LVH}$ in an African-American population. ${ }^{(8)}$

In the presence of $\mathrm{HF}$, there are particular abnormalities identified from the ECG that can characterize the syndrome, such as conduction abnormalities (e.g. bundle branch blocks, high amplitude $\mathrm{R}$ waves indicating hypertrophy and wide QRS complexes). ${ }^{(7)}$ Indeed, one recent report from predominantly Caucasian populations living in high income countries indicated that over $90 \%$ of those with HF had an abnormal ECG. ${ }^{(7)}$

\section{STUDY AIMS}

The primary aim of this study was to undertake a preliminary analysis of ECG data derived from a large cohort of Black African patients with HF (confirmed by echocardiography) within the 2006 Heart of Soweto study cohort to determine the concurrent prevalence and type of major and minor ECG abnormalities. Ultimately, these data will be used to evaluate the role of I 2-lead ECGs in detecting previously undiagnosed HF in this setting.

\section{METHODS}

\section{Participants \& Setting}

As previously described in more detail, the Heart of Soweto study was established to investigate the prevalence and incidence of CVD within the townships of Soweto, South Africa. ${ }^{(2)}$ As part of this study, we have systematically examined the volume, spectrum and pattern of clinical presentations in those attending the Cardiology Unit of the Chris Hani Baragwanath Hospital. The hospital provides health care to the estimated I to 1.5 million predominantly black Africans residing in the adjacent townships of Soweto.The study was approved by the hospital's Ethics of Human Research Committee.

As described in our index report from the Heart of Soweto study,(II) all first-time patients diagnosed with HF presenting to Chris Hani Baragwanath Hospital between the I st January to 3 I st December 2006 were subject to a combination of echocardiographic assessment and review by a supervising cardiologist, in either the cardiac clinic or Coronary Care Unit. Only patients with confirmed HF (see diagnostic criteria below) were included in the study. This comprises a total of 844 patients with a combination of symptoms of HF and objective evidence of cardiac dysfunction (systolic and/or diastolic) at rest. As indicated above, all patients included in this report were subject to echocardiographic assessment with detailed assessment of ventricular function, valvular integrity and function and regional wall abnormalities (specific measurements were available in all but $4 \mathrm{I}$ cases [5\%]). Specifically, two-dimensional targeted M-mode echocardiography with Doppler colour flow mapping was performed using a Hewlett Packard Sonos 5500 echocardiograph attached to a 2.5 or $3.5 \mathrm{MHz}$ transducer. All echocardiographic procedures were undertaken by trained operators and measurements were made according to the American Society of Echocardiography guidelines. ${ }^{(12)}$ Diastolic mitral flow was assessed by pulsed-wave Doppler echocardiography from the apical four-chamber view. The E wave deceleration time was also measured as the interval between the peak early diastolic velocity and the point at which the steepest deceleration slope is extrapolated to the zero line.

A detailed cardiac assessment was performed by a qualified or trainee Cardiologist to document lung and heart sounds and other clinically significant signs and symptoms. Functional status was also assessed to determine New York Heart Association Classification of dyspnea. ${ }^{(13)}$

\section{Clinical classification}

All cases were reviewed by SS and KS. These are based on European Society of Cardiology current guidelines ${ }^{(13)}$ and the recently published European Heart Survey. ${ }^{(14)}$ Based on the above process, the research team documented a clinical diagnosis for every individual included in the registry and captured close to 100\% of their basic socio-economic and clinical profile from the standardized interview and assessments. The following forms of HF were identified as part of the clinical review process:

\section{- Left ventricular systolic dysfunction}

Left ventricular ejection fraction (LVEF) $<45 \%$.

\section{- Diastolic dysfunction}

Classified based on E/A ratio and deceleration time according to generally accepted criteria. ${ }^{(12)}$

\section{Dilated cardiomyopathy}

LVEF $\leq 45 \%$ and Left Ventricular Dimension in Diastole $>55 \mathrm{~mm}$ with global regional wall motion abnormality.

\section{- Ischemic cardiomyopathy}

LVEF $<45 \%$, confirmed diagnosis of CAD and regional wall motion abnormality.

\section{- Hypertensive heart failure}

Blood pressure of $>180 / 100 \mathrm{mmHg}$ accompanied by symptoms of HF (dyspnea and tachycardia), increased LV septal thickness (> $1.3 \mathrm{~mm}$ ), diastolic dysfunction and/or systolic dysfunction (LVEF $<45 \%$ ) by echocardiography. 


\section{Valvular heart failure}

Secondary to primary underlying valve disease (e.g. rheumatic heart disease or degenerative valve disease) as opposed to underlying valvular dysfunction (e.g. mitral regurgitation) due to underlying cardiac dysfunction/failure.

\section{Right heart failure}

Secondary to right-sided pathophysiology with increased jugular venous pressure and liver size, tricuspid regurgitation and elevated right ventricular systolic pressure $>35 \mathrm{mmHg}$. Usually accompanied by peripheral oedema as unique or concomitant to left ventricular failure.

\section{Other forms of heart failure}

That includes congenital heart disease with delayed diagnosis made only in adulthood, hypertrophic obstructive cardiomyopathy, endomyocardial fibrosis, sub-mitral aneurysm, and secondary to infectious pericardial effusion.

On confirmation of the presence of $\mathrm{HF}$, detailed clinical records were completed, including demographic data (age, race and sex with sociodemographic profile) and clinical data (encompassing blood pressure, anthropometric measurements, self-reported cardiac risk factors, functional status for angina and breathlessness). All patients were given a unique identifying code to allow further data analyses using the clinica registry. The total number of new "de novo" HF cases seen by the Cardiology Unit of the Chris Hani Baragwanath Hospital in the calendar year 2006 was 844. Of these, 756 (90\%) had a documented I2-lead ECG that was entered into the clinical registry with a hard copy available for detailed analyses.

\section{ECG analysis}

A 12-lead resting ECG was performed by a trained technician and analysed by blinded reviewers (clinical cardiac nurses with advanced training) using the Minnesota Code Classification system. ${ }^{(15)}$ Some had undertaken post-graduate diplomas in cardiac nursing and all worked in clinical nursing positions within cardiology. Two cardiac trained nurses (SS \& GL) then independently verified these ECGs. The code allows systematic classification of $\mathrm{Q}$ and $\mathrm{QS}$ patterns, axis deviation, $\mathrm{R}$ waves, ST depression and elevation and T wave changes, along with conduction abnormalities in both atria and ventricles. ${ }^{(15,16)}$ In addition to the Minnesota code, we also examined $\mathrm{P}$ wave changes (that is, left, right and biatrial changes that reflect atrial hypertrophy).

The abnormalities detected by the Minnesota code were pooled into major and minor abnormalities and separate analyses were performed
TABLE I: Definition of ECG abnormalities and corresponding Minnesota codes ${ }^{(14)}$

\begin{tabular}{|c|c|}
\hline ECG Abnormality & Minnesota Code \\
\hline \multicolumn{2}{|l|}{ Major abnormalities } \\
\hline $\begin{array}{l}\text { Q and QS patterns (indicating myocardial } \\
\text { infarction) }\end{array}$ & Code $I_{1-2}$ \\
\hline $\begin{array}{l}\text { ST depression (Anterolateral, posterior, } \\
\text { anterior) }\end{array}$ & Codes $I_{1-2}$ \\
\hline Negative $T$ waves & Codes $V_{1-2}$ \\
\hline $\begin{array}{l}\text { Heart block } \\
\text { (Wenckebach, Partial, Mobitz type II, } \\
\text { complete heart block) }\end{array}$ & Codes $\mathrm{VI}_{1-2}$ \\
\hline $\begin{array}{l}\text { Ventricular conduction defects } \\
\text { (Bundle branch block - complete or } \\
\text { intermittent) }\end{array}$ & Codes VII \\
\hline $\begin{array}{l}\text { Arrthymias } \\
\text { (Premature atrial or ventricular beats, } \\
\text { atrial/ventricular fibrillation or flutter) }\end{array}$ & Codes VIIII-3 \\
\hline \multicolumn{2}{|l|}{ Minor abnormalities } \\
\hline Borderline Q wave abnormalities & 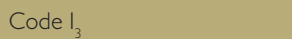 \\
\hline Axis Deviation (left or right) & Codes $\|_{1-2}$ \\
\hline High amplitude R waves & Code III \\
\hline ST segment downward sloping & Code $\mathrm{IV}_{3}$ \\
\hline Flattened T waves & $\mathrm{CodeV}_{3}$ \\
\hline Low QRS voltage & Code $\mid X_{1}$ \\
\hline \multicolumn{2}{|l|}{ Other abnormalities } \\
\hline Ischemic findings & Codes $I_{3}$ or $\mathrm{IV}_{1-3}$ or $\mathrm{V}_{1-3}$ or $\mathrm{V} \mathrm{II}_{1}$ \\
\hline ST depression & Codes $\mathrm{IV}_{1-3}$ \\
\hline Arrhythmias & Codes VIII or $\left.\mathrm{VII}\right|_{1-2,4}$ \\
\hline Bundle branch blocks & Codes VIII-2,4 \\
\hline Left ventricular hypertrophy & Codes III, and $\left(\mathrm{IV}_{1-3}\right.$ or $\left.\mathrm{V}_{1-3}\right)$ \\
\hline Left axis deviation & Codes $\|_{1}$ \\
\hline
\end{tabular}

for ST segment depression, arrhythmias or AV blocks, bundle branch blocks and left axis deviation. The rationale for major and minor abnormalities was based primarily on a previous study by De Bacquer and colleagues ${ }^{(17)}$ and the lack of consenses in other studies regarding the classification of ECG abnormalities..$^{(9.10)(18-20)}$ Also, there are potential clinical advantages in dividing ECG abnormalities into major and minor changes. The relevant Minnesota codes for each abnormality group are presented in Table I.

Major abnormalities were defined as those with $\mathrm{Q}$ wave abnormalities, ST segment depression, T wave inversion, complete or second degree $\mathrm{AV}$ block, complete left or right bundle branch block (LBBB, RBBB), frequent premature beats, atrial fibrillation or flutter. Those with minor abnormalities were defined as borderline $Q$ waves, left or right axis deviation, high amplitude R waves, borderline ST segment depression, T wave flattening and low QRS voltage. Sinus tachycardia was defined as a rate of $>100 \mathrm{bpm}$ and sinus bradycardia $<50 \mathrm{bpm}$. 


\section{Data analysis}

Simple frequency and correlational analyses of ECG abnormalities according to the underlying type of HF were performed using SPSS version 13

\section{RESULTS}

\section{Clinical \& Demographic Profile}

Of the 756 patients with a confirmed diagnosis of HF in the study cohort, 57\% ( $n=478)$ were female, 88\% $(n=665)$ were black African and their mean age was 55 years ( \pm 16 years). More than one recognizable HF antecedent was evident in over $90 \%$ of the sample, with $70 \%$ having hypertension, $47 \%$ being smokers and 34\% being obese.The mean LVEF was $45 \pm 18 \%$ with $27 \%$ presenting with diastolic dysfunction (the majority had preserved systolic function). Five predominant forms of HF were identified in this cohort: dilated cardiomyopathy $(C M O)(n=251,35 \%)$, hypertensive HF ( $n=242,33 \%)$, right HF ( $n=193,27 \%)$, valvular HF $(n=49,8 \%)$ and ischemic CMO $(n=65,9 \%)$. Black Africans were far more likely to present with a dilated CMO (OR 4.39, 95\% Cl 2.4I to 8.00) but far less likely to present with ischemic CMO (OR 0.1 I, 95\% Cl 0.07 to 0.19). Patients with dilated CMO (7\% and 12\%) and right HF (10\% and 40\%) were most likely to have moderate to severe mitral or tricuspid regurgitation.

\section{Major and minor abnormalities}

When applying Minnesota coding of each patient's I2-lead ECG, major ECG abnormalities (refer to Table I) were recorded in 91\% of HF patients. The most notable major abnormality was negative $T$ waves in $57 \%$ of ECGs ( $n=428$ ) - refer to Figure I. Other common abnormalities were ST segment depression in $21 \%(n=159)$ followed by $Q$ waves in $18 \%(n=135)$. Only $3 \%$ of ECGs were normal.

Most ECGs indicated that patients were in sinus rhythm $(n=475,63 \%)$ or sinus tachycardia ( $n=198,26 \%)$. A small number of patients $(n=13$, $2 \%$ ) had a heart rate $<50$ bpm. A further 4 I patients (5\%) showed left anterior hemiblock and eight ECGs demonstrated intraventricular block. A close examination of $\mathrm{P}$ wave morphology revealed a left atrial abnormality in 100 patients (13\%) and a further 93 ECGs (I2\%) demonstrated right atrial abnormality. Biatrial abnormality was evident in 60 ECGs (8\%). In relation to the examination of AV conduction defects, three patients had an ECG suggesting a complete heart block, one patient a Mobitz heart block and two patients had pacemakers with appropriate capture. Three ECGs revealed a shortened PR interval, while 34 (4.4\%) revealed a prolonged PR interval. A total of 64 patients (8\%) were in atrial fibrillation or atrial flutter. Of these,
52 ECGs demonstrated persistent AF / persistent atrial flutter, with the remainder demonstrating intermittent $\mathrm{AF}$.

A total of $97 \%$ of ECGs demonstrated minor ECG abnormalities (refer to Figure 2). Of these, the most common were axis deviation and high $R$ wave amplitude ( $n=232,31 \%$ ), followed by flattened T waves in $24 \%$ of ECGs $(n=179)$. Borderline ST segment depression (downward sloping ST segments) and borderline Q waves were observed in 48 ECGs (6\%). Low QRS voltage was also recorded in 5\% of ECGs $(n=37)$.

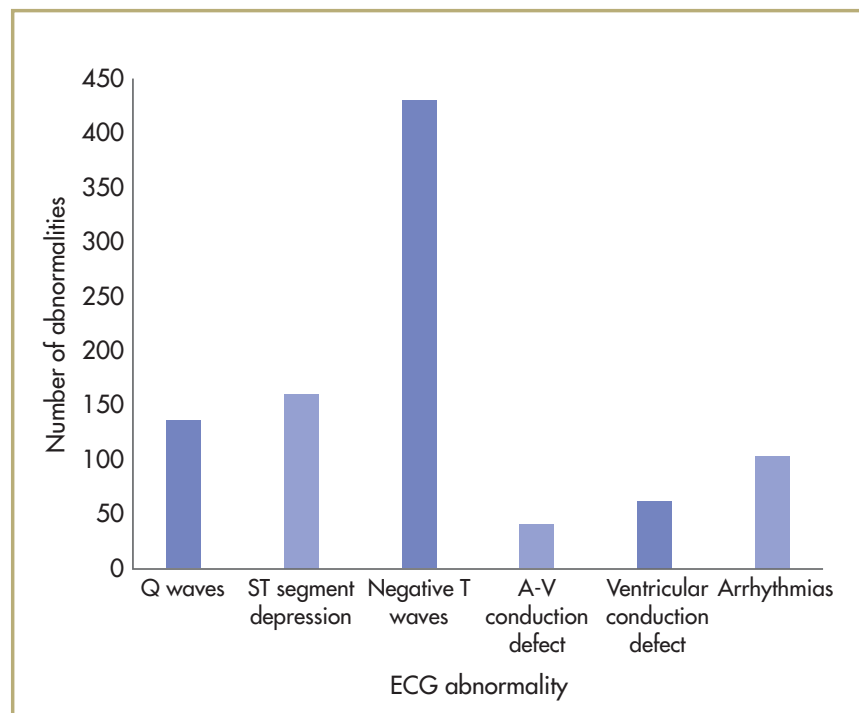

FIGURE I: Frequency of major ECG abnormalities in 756 predominantly blackAfrican patients with $\mathrm{HF}$

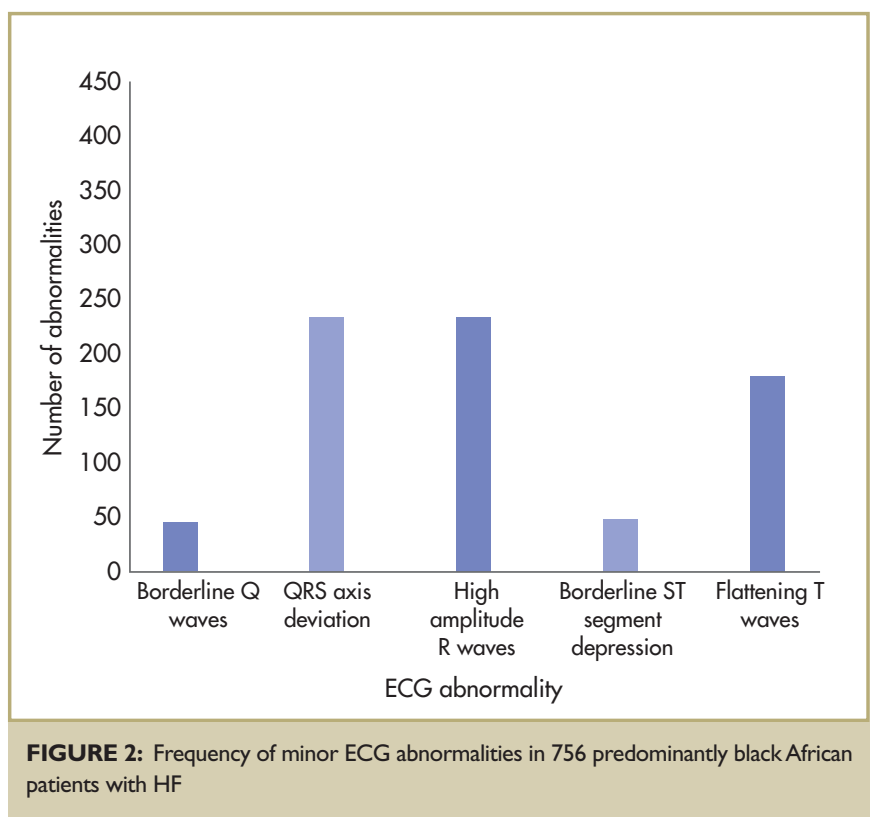




\section{ECG ABNORMALITIES IN HEART FAILURE}

\section{ECG characteristics indicating possible HF}

Specific characteristics that can be detected on a I2-lead ECG that are an indicator of HF include left ventricular hypertrophy $(\mathrm{LVH})$, bundle branch blocks and axis deviation. Given that LVH is often associated with the development of HF (particularly in hypertensive individuals), it was interesting to note that ECG evidence of LVH was coded in I5\% of this patient cohort $(n=|| 0)$. This was based on a Minnesota coding of isolated high amplitude R wave (code III) and either ST elevation (code $\mathrm{IV}_{1-3}$ ) or $\mathrm{T}$ wave changes (code $\mathrm{V}_{1-3}$ ). . $^{(15)}$ Overall, LVH was observed in the ECGs of 54 patients with a dilated CMO (49\%), 35 patients with hypertensive $\mathrm{CMO}$ (32\%) and 22 of those with right HF (20\%). In contrast, only four patients diagnosed with an ischemic CMO (3.6\%) had ECG evidence of LVH ( $p<0.00$ I for the comparison to all other diagnostic groups).

Figure 3 highlights the abnormalities typically observed on an ECG in a person with heart failure. It highlights tall R waves, ST segment changes and inverted $T$ waves. All of these characteristics substantiate the probability of underlying LVH.

Bundle branch blocks are associated with HF and ventricular dysynchrony resulting in a decrease in cardiac output, adding to the already overburdened heart. With respect to ventricular conduction defects, (namely bundle branch blocks), a total of 32 ECGs (4\%) had complete LBBB and II (1.5\%) had RBBB. A further I3 (1.8\%) had incomplete/ intermittent RBBB and 14 (1.8\%) had incomplete left bundle branch blocks.

Although the majority of ECG axes were normal $(n=51$ I), I 30 (I7\%) indicated a right axis deviation and 107 (14\%) a left axis deviation (see Table 2).

\section{DISCUSSION}

Examination of the ECG data on de novo HF presentations in the Heart of Soweto study revealed a high prevalence of ECG abnormalities. Specifically, we found that Minnesota coding of I2-lead ECGs revealed major and minor abnormalities in $91 \%$ and $97 \%$ of patients, respectively. As such, only 3\% of ECGs were normal. Other ECG characteristics that are associated with HF (although these can also present with other pathologies) were shown in $8 \%$ of ECGs demonstrating bundle branch block, $15 \%$ with characteristics of LVH and a further $31 \%$ of ECGs had axis deviation. Thus the ECG plays a potentially important role in identifying abnormalities that are connected to a diagnosis of HF in

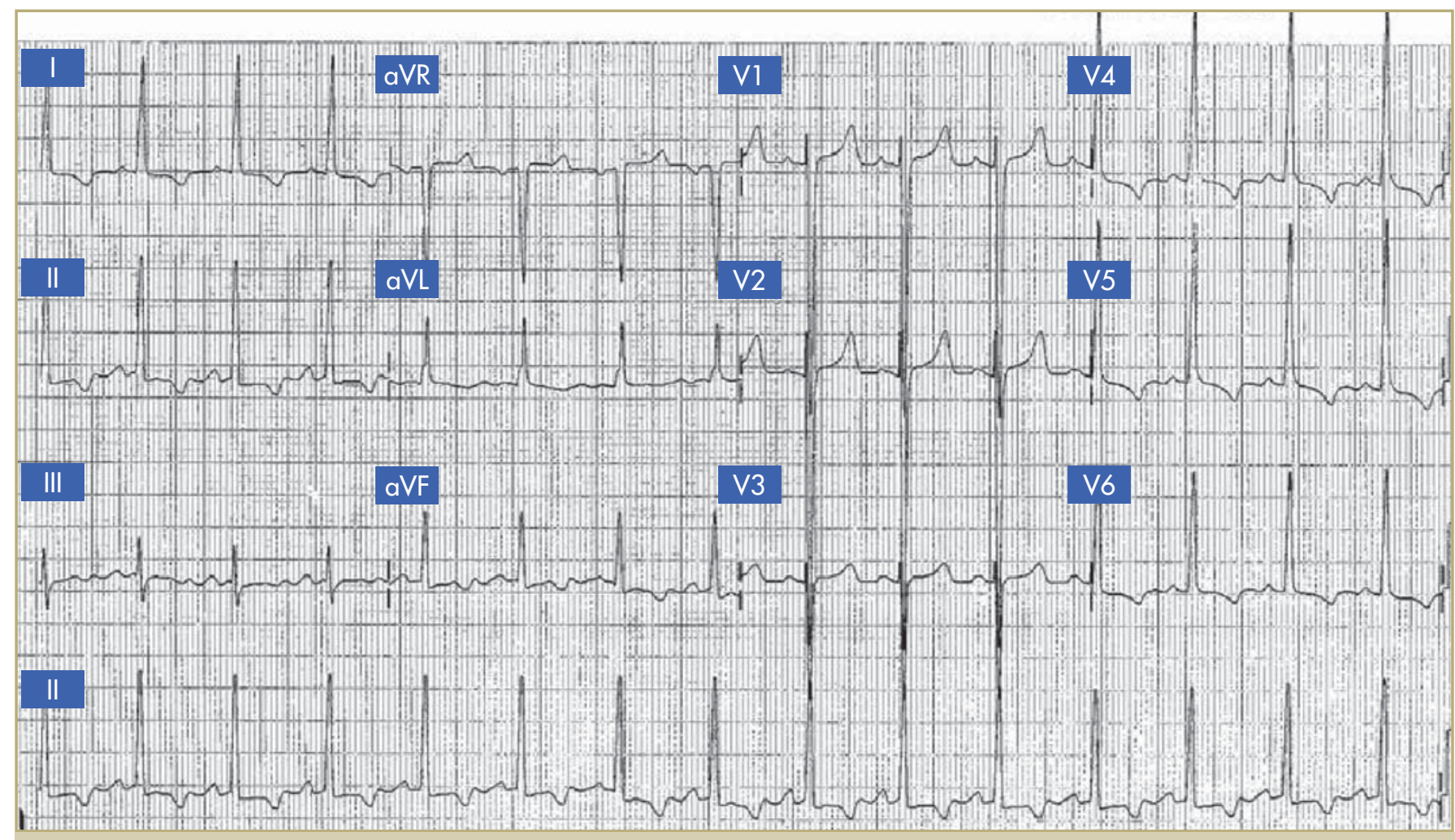

FIGURE 3: An example of a I2-lead electrocardiogram from a 45-year-old black South African female with hypertensive cardiomyopathy illustrating tall R waves, ST segment and T wave changes in the precordial leads (VI-V6). 
TABLE 2: Axis and rhythm characteristics in this HF cohort

\begin{tabular}{|l|r|}
\hline \multicolumn{1}{|c}{ ECG Abnormality } & Number of cases (\%) \\
\hline Axis & $511(68 \%)$ \\
\hline Normal axis & $107(14 \%)$ \\
Left axis deviation & $130(17 \%)$ \\
Right axis deviation & $2(0.2 \%)$ \\
Extreme deviation & $3(0.3 \%)$ \\
Indeterminate & \\
\hline Rhythm & $475(63 \%)$ \\
\hline Sinus Rhythm & $198(26 \%)$ \\
Sinus Tachycardia & $13(1.7 \%)$ \\
Sinus Bradycardia & $52(6.8 \%)$ \\
Atrial fibrillation/flutter (persistent) & $3(0.3 \%)$ \\
Heart block & $5(0.6 \%)$ \\
\hline Junctional arrhythmia & \\
\hline \hline
\end{tabular}

this clinical setting of predominantly urban dwelling black Africans in South Africa.

In the present study, the majority of ECGs were abnormal. This was not surprising, however, since HF is the end result of poor cardiovascular health, which other conditions predispose the development of.The high number of abnormalities is in total agreement with previous studies that detected anomalies in the majority of ECGs of HF patients, ${ }^{(17,18)}$ with one study reporting abnormal ECGs in $97 \%$ of patients. ${ }^{(17)}$ The general consensus is that the ECG, in conjunction with patient symptoms, has an important role in guiding therapy and provides a basis for furtherinvestigation, that most likely being echocardiography. ${ }^{(18,19)}$ It is also important to recognize that abnormal ECG findings strongly suggest the presence of abnormal cardiac functioning which, if left unmonitored and untreated, can lead to a diagnosis of HF.

Whilst a large number of HF patients had abnormal ECGs, more specific indications of HF from the ECG were detected in a smaller number of individuals. Overall, any ECG characteristic associated with HF (LVH, bundle branch blocks and axis deviation) were found in a total of 362 patients (48\%). In comparison to the few studies in the literature that report the proportions of HF patients with ECG characteristics of HF, the number of LBBB observed on the ECGs in the current study was approximately one-third of that reported in other studies. ${ }^{(20,21)}$ This discrepancy may be explained by the multiplicity of etiologies that can drive the development of HF (in this context from chronic hypertension to right heart failure to idiopathic CMOs). As such, in this cohort, in contrast to studies undertaken in high income countries where atherosclerotic disease predominates, ischemic $\mathrm{CMO}$ was the exception rather than the rule. ${ }^{(1)}$
There are many choices in determining an ECG classification tool. We chose to apply the Minnesota code to detect ECG abnormalities because it has been well validated, it is applicable to most populations compared to other indices ${ }^{(8)}$ and, most importantly, it encapsulates the major ECG abnormalities that are important in describing an individual's electrophysiological function. A recent systematic review concluded that alternative electrocardiography indices deemed useful for detecting LV hypertension (Sokolow-Lyon index, Cornell voltage index, Cornell product, Gubner and Romhilt-Estes scores) suffered from poor accuracy. ${ }^{(9)}$ Also, the amplitude of the signal voltage on ECGs poorly predicted $\mathrm{LVH}^{\left({ }^{(10)}\right.}$ Finally, the Minnesota code has commonly been employed as a "prognostic" tool to determine long-term health outcomes and is associated with all-cause and CVD mortality. ${ }^{(8-10,17,22-24)}$

As an alternative marker of underlying ventricular dysfunction, brain natriuretic peptide (BNP) is a biochemical marker also used to detect $\mathrm{HF}$ and recommended to be used in conjunction with the ECG. ${ }^{(6)} \mathrm{BNP}$ is strongly related to the diagnosis of chronic heart failure ( $\mathrm{CHF}$ ), although the results have been less accurate in elderly patients and in women. ${ }^{(25,26)}$ A BNP of $>100 \mathrm{pg} / \mathrm{mL}$ has been shown to provide relatively high sensitivity (89\%) and specificity (73\%) for diagnosing HF. ${ }^{(7,27)}$ From a cost perspective, the unit cost of BNP is disproportionately more expensive compared to ECG but, due to differences in sensitivity and specificity in detecting HF, BNP becomes equally cost-effective as ECG. ${ }^{(7)}$ These authors report that the gold standard strategy of traditional echocardiography alone was least cost-effective for screening, supporting that the ECG (or BNP) can be used cost-effectively for screening. Acknowledging the financial cost of treating and managing HF, the ECG clearly plays an important role. It may play an even greater role in low to middle income countries with a greater pool of individuals with more advanced forms of HF and where the cost constraints of using expensive bio-markers is more limited.

Pending further analyses to determine the sensitivity, specificity and positive predictive accuracy of the 12 -lead ECG relative to gold standard echocardiography, these preliminary data indicate a potential role for the 12-lead ECG in any systematic screening program for HF in Soweto (and other communities). In the Heart of Soweto study, echocardiographic assessment was performed in all patients to determine ventricular function and functional abnormalities. However, the purpose of this paper was to highlight the ECG abnormalities independently of echocardiography. A correlation of the two measures will be performed in the near future. 
This study has a number of limitations that require comment. For example, one of the disadvantages of a I2-lead ECG is that it is relatively brief in capturing a 10-second tracing of the heart's electrical activity, therefore risking missing transient changes. However, it is markedly advantageous over a 3-lead ECG, which provides very limited information. An important aspect is that the ECGs were not performed in isolation but in conjunction with other clinical measurements, thus allowing an in-depth assessment of an individual's clinical status. Although the 12-lead ECG is beneficial in aiding diagnoses and as a useful preliminary screening tool, the gold standard for a confirmatory HF diagnosis is the echocardiogram. One shortcoming of the Minnesota code is that it does not examine $P$ wave abnormalities that indicate atrial enlargements and are associated with $\mathrm{P}$ mitrale (left atrial hypertrophy), P pulmonale (right atrial hypertrophy) or biphasic $P$ waves (suggesting combined atrial hypertrophy). The detection of $\mathrm{P}$ wave abnormalities is considered important, as they may indicate untreated or poorly treated hypertension or represent some other unconfirmed pathology. To overcome this inconsistency in future applications of Minnesota coding of ECGs, we have decided to incorporate an examination of $\mathrm{P}$ wave abnormalities into our investigations.

Despite these limitations, and given the potential for the future rise of HF in Africa within the constraints of limited public health budgets, these preliminary data suggest that the 12-lead ECG has the ability to play a central role in identifying abnormal characteristics associated with conditions such as HF. As such, our detailed examination of a large group of previously undiagnosed patients with HF in the Heart of Soweto study revealed many identifiable abnormalities. In addition to reconfirming the clinical utility of this cheap and practical diagnostic tool, these data highlight its potential role in screening for heart disease, particularly in low income communities.

\section{REFERENCES:}

I. Stewart S, Maclntyre K, Hole DJ, Capewell S, McMurray J]. More 'malignant' than cancer? Five-year survival following a first admission for heart failure. Eur J Heart Fail. 200 I Jun;3(3):315-22.

2. Stewart S, Wilkinson D, Becker A, Askew D, Ntyintyane L, McMurray JJ, Sliwa K. Mapping the emergence of heart disease in a black, urban population in Africa:the Heart of Soweto Study. Int J Cardiol. 2006 Mar 22; 108(1): I 0 I-8. Epub 2006 Feb 7.

3. Yusuf S, Reddy S, Ounpuu S, Anand S. Global burden of cardiovascular diseases. Part I: General considerations, the epidemiologic transition, risk factors and impact of urbanization. Circ 200 I; 104: 2746-53.

4. Sliwa K, Damasceno A, Mayosi BM. Cardiomyopathy in Africa. Circ 2005; 1 I 2 : 3577-83.

5. Akinboboye $\mathrm{O}$, Idris $\mathrm{O}$, Akinboboye $\mathrm{O}$, Akinkugbe $\mathrm{O}$. Trends in coronary artery disease and associated risk factors in sub-Saharan Africans. J Hum Hypertens. 2003 Jun; 17(6): $381-7$.

6. Troughton RW, Richards M. B-type natriuretic peptides: applications to heart failure management in 2005. Int Med J 2005; 35: 377-79.

7. Galasko GIW, Barnes SC, Collinson P, Lahiri A, Senior R. What is the most cost-effective strategy to screen for left ventricular systolic dysfunction: natriuretic peptides, the electrocardiogram, hand-held echocardiography, traditional echocardiography, or their combination? Eur Heart J 2006; 27: 193-200.

8. Okin PM, Wright JT, Nieminen MS, Jern S, Taylor AL, Phillips R, Papademetriou V, Clark LT, Ofili EO, Randall OS, Oikarinen L, Viitasalo M, Toivonen L, Julius S, Dahlöf B, Devereux RB. Ethnic differences in electrocardiographic criteria for left ventricular hypertrophy: the LIFE study. Losartan Intervention For Endpoint. Am J Hypertens. 2002 Aug; |5(8):663-7|.

9. Pewsner D, Juni P, Egger M, Battaglia M, Sundstrom J, Bachmann LM. Accuracy of electrocardiography in diagnosis of left ventricular hypertension: systematic review. BMJ 2007; doi: I0.1 I36.bmj.39276.636354.AE

10. Morrison I, Clark E, Macfarlane PW. Evaluation of the electrocardiographic criteria for left ventricular hypertrophy. Anadolu Kardiyol Derg 2007; 7 Suppl I: 159-163.

II. Sliwa K, Wilkinson D, Hansen C, Ntyintyane L, Tibazarwa K, Becker A, Stewart S. Spectrum of heart disease and risk factors in a black urban population in South Africa (the Heart of Soweto Study): a cohort study. The Lancet 2008. In press.

12. Sahn DJ, DeMaria A, Kisslo J, Weyman A. Recommendations regarding quantitation in M-mode echocardiography: results of a survey of echocardiographic measurements. Circulation. 1978 Dec;58(6): 1072-83.

13. Swedberg K, Cleland J, Dargie H, Drexler H, Follath F, Komajda M,Tavazzi L, Smiseth OA, Gavazzi A, Haverich A, Hoes A, Jaarsma T, Korewicki J, Lévy S, Linde C, Lopez-Sendon JL. Nieminen MS, Piérard L, Remme W]; Task Force for the Diagnosis and Treatment of Chronic Heart Failure of the European Society of Cardiology. Guidelines for the diagnosis and treatment of chronic heart failure: executive summary (update 2005):The Task Force for the Diagnosis and Treatment of Chronic Heart Failure of the European Society of Cardiology. Eur Heart J. 2005 Jun;26(I I): I I I5-40. Epub 2005 May 18. No abstract available.

14. Nieminen MS, Brutsaert D, Dickstein K, Drexler H, Follath F, Harjola V, Hochadel M, Komajda M, Lassus J, Lopez-Sendon JL, Ponikowski P, and Tavazzi $L$ on behalf of the EuroHeart Survey Investigators. EuroHeart Failure Survey II (EHFS II): A survey on hospitalized acute heart failure patients: description of population. Euro Heart J 2006: 27:2725-2736

15. Prineas R, Crow R, Blackburn H. The Minnesota Code Manual of Electrocardiographic Findings. John Wright-PSG, Inc. Littleton, MA, June 1982.

16. Blackburn H, Keys A, Simonson E et al. The electrocardiogram in population studies. A classification system. Circ 1960; 21: I 160-75.

17. De Bacquer D, De Backer G, Kornitzer M, Blackburn H. Prognostic value of ECG findings for total, cardiovascular disease, and coronary heart disease death in men and women. Heart 1998; 80: 570-577. 
18. Khan NK, Goode KM, Cleland JG, Rigby AS, Freemantle N, Eastaugh J, Clark AL, de Silva R, Calvert MJ, Swedberg K, Komajda M, Mareev V, Follath F; EuroHeart Failure Survey Investigators. Prevalence of ECG abnormalities in an international survey of patients with suspected or confirmed heart failure at death or discharge. Eur J Heart Fail. 2007 May;9(5):491-50।. Epub 2007 Jan 9.

19. Talreja D, Gruver C, Sklenar J, Dent J, Kaul S. Efficient utilization of echocardiography for the assessment of left ventricular systolic function. Am Heart J 2000; 139: 394-8.

20. Lee SJ, McCulloch C, Mangat I, Foster E, De Marco T, Saxon LA. Isolated bundle branch block and left ventricular dysfunction. J Card Fail 2003; 9 (2): 87-92.

21. Baldasseroni S, Opasich C, Gorini M, Lucci D, Marchionni N, Marini M, Campana C. Perini G, Deorsola A, Masotti G, Tavazzi L, Maggioni AP; Italian Network on Congestive Heart Failure Investigators. Left bundle-branch block is associated with increased I-year sudden and total mortality rate in 5517 outpatients with congestive heart failure: a report from the Italian network on congestive heart failure. Am Heart J. 2002 Mar; | 43(3):398-405.

22. Strom Moller CS, Zethelius B, Sundstrom J, Lind L. Persistent ischemic ECG abnormalities on repeated ECG examination have important prognostic value for cardiovascular disease beyond established risk factors: a population-based study in middle-aged men with up to 32 years follow-up. Heart; 93: I 104-1 1 I0

23. Jimenez-Corona A, Nelson RG, Sievers ML, Knowler WC, Hanson RL, Bennett PH. Electrocardiographic abnormalities predict deaths from cardiovascular disease and ischemic heart disease in Pima Indians with type 2 diabetes. Am Heart J; I5I (5): 1080-1086.

24. Greenland P, Xie X, Liu K, Colangelo L, Liao Y, Daviglus ML, Agulnek AN, Stamler J. Impact of minor electrocardiographic ST-segment and/orT-wave abnormalities on cardiovascular mortality during long-term follow-up. Am J Cardiol; 9 I (9) : 1068- 1074.

25. Knudsen CW, Riis JS, Finsen AV, Eikvar L, Müller C, Westheim A, Omland T. Diagnostic value of a rapid test for B-type natriuretic peptide in patients presenting with acute dyspnea: effect of age and gender. Eur J Heart Fail. 2004 Jan;6(I):55-62.

26. Loke I, Squire IB, Davies JE, Ng LL. Reference ranges for natriuretic peptides for diagnostic use are dependent on age, gender and heart rate. Eur J Heart Fail. 2003 Oct;5(5): 599-606.

27. Steg PG, Joubin L, McCord J, Abraham WT, Hollander JE, Omland T, Mentré F, McCullough PA, Maisel AS. B-type natriuretic peptide and echocardiographic determination of ejection fraction in the diagnosis of congestive heart failure in patients with acute dyspnea. Chest. 2005 Jul; | 28( I):21-9. 